\section{Negative incentive contrast effects with saccharin vs sucrose and partial reinforcement *}

\author{
LAWRENCE WEINSTEIN $\dagger$ \\ University of Melbourne, Parkville, Vic toria 3052, Australia
}

Thirty male hooded rats received seven sessions of barpress training with sucrose (16\% or $4 \%$ ) and saccharin $(.10 \%$ or $1.5 \%)$ as reward, followed by a shift from $16 \%$ to $4 \%$ sucrose and $.10 \%$ to $1.5 \%$ saccharin. Four dependent measures indicated that concentrations of sucrose and saccharin that produced equivalent levels of performance produced equivalent negative contrast effects. In Experiment 2, 18 male hooded rats received four sessions of barpress training with $(.10 \%$ or $1.5 \%)$ saccharin under an FR 7 schedule of reinforcement followed by a shift from $.10 \%$ to $1.5 \%$. Four of the five measures indicated negative contrast effects. Confounding inherent in the use of solid food or sucrose does not appear to account for negative incentive contrast effects.

Negative incentive contrast effects are obtained when the performance of Ss exposed to a decrease in amount of reinforcement drops significantly below the performance of control Ss exposed only to the single lower reward magnitude. Studies typically use solid food or sucrose solution as incentive. A decrease in amount of solid food or concentration of sucrose is confounded with a difference in body weight. Dunham \& Kilps (1969) have demonstrated that preshift training under a $32 \%$ concentration of sucrose solution significantly increases body weight as compared to a $4 \%$ concentration; the difference in body weight is confounded with a shift in reinforcement conditions. This source of confounding may be eliminated by using saccharin solutions as incentives. Collier \& Novell (1967) have demonstrated that consumption of a $.01 \%$, $.03 \%, .30 \%, .90 \%$, or $2.7 \%$ saccharin solution does not alter body weight or subsequent food ingestion compared to a $.10 \%$ saccharin solution.

Hulse (1962) and Vogel, Mikulka, \& Spear (1968) decreased the concentration of a constant-volume saccharin solution and failed to obtain negative contrast effects. This may be because they selected preshift concentrations not conducive to the production of negative contrast effects. Many studies have shown that the magnitude of negative contrast effects is a positive function of the amount of reward

\footnotetext{
*This research, conducted at Queen's University, Kingston, Ontario, was presented at the 1969 Eastern Psychological Association meeting in Philadelphia.
}

$\div$ Requests for reprints should be sent to Lawrence Weinstein, Department of Psychology, University of Melbourne, Parkville, Victoria 3052, Australia. reduction (e.g., Di Lollo \& Beez, 1966). Hulse (1962) decreased the concentration of a saccharin solution from $1 \%$ to $.1 \%$ following training on a FR 8 schedule in an operant-conditioning chamber using the consummatory response. Vogel et al (1968) reduced the concentration of a saccharin solution from $.10 \%$ to $.01 \%$ following training on CRF in an operant-conditioning box using the consummatory response. Quite possibly larger reductions in incentive magnitude would produce negative contrast effects. Collier \& Novell (1967) have shown that the preference curve for saccharin is an inverted-U-shaped function with the maximum intake at $.10 \%$ and the minimum from $1.5 \%$ to $2.7 \%$. Their data indicate that these concentrations $(.10 \%$ and $1.5 \%-2.7 \%$ ) would probably produce greater disparities in behavior than either set of concentrations selected by Hulse or Vogel et al. Hence, a shift from $.10 \%$ to $1.5 \%-2.7 \%$ would constitute a larger change in reward magnitude than the procedures used by Hulse (1962) and Vogel et al (1968) and would therefore be more likely to result in negative contrast effects. If negative contrast effects are not obtained with an appropriate change in the concentration of a saccharin solution, then all previous demonstrations of negative contrast effects would appear to be due to confoundings inherent in the use of solid food or sucrose. Experiment 1 examined changes in incentive under saccharin and sucrose.

Studies that have examined the effects of decreasing incentive magnitude following training on a partial schedule of reinforcement characteristically use solid food or sucrose. Hulse (1962) used saccharin and obtained a measure of incentive decrease in the relative absence of confounding factors. He obtained no evidence of negative contrast effects. As already mentioned, this may be due to his selection of preshift concentrations. If negative contrast effects are not obtained with an appropriate shift in the concentration of a saccharin solution, then it would appear that all demonstrations of negative contrast effects under partial reinforcement are the result of confoundings inherent in the use of solid food or sucrose. Experiment 2 examined changes in incentive under saccharin and a partial schedule of reinforcement.

\section{EXPERIMENT 1}

Subjects

The Ss were 30 experimentally naive hooded rats, 75.105 days old at the start of the experiment: they were housed in individual cages.

\section{Apparatus}

The conditioning chamber had plywood side walls, Plexiglas top, back, and door, and a grid floor; it measured $12 \times 10 \times 10$ in.

A rectangular hole, $1 / 2$ in. high $\times 2$ in. kong, was cut in the side of the cage 5 in. above the floor so that the bar could be inserted $1 / 2 \mathrm{in}$. into the cage. Three inches below and 2 in. to the side of the bar, a circular hole, 1 in. in diam, was cut in the wall. Through this opening $S$ had access to the liquid magazine.

The liquid used was presented to $S$ by a dipper-type magazine driven by a motor. A metal drinking tube, attached to a 3-ft piece of plastic tubing which connected to a plastic cylinder graduated in ccs was mounted $1 \mathrm{in}$. outside the circular hole in the wall so that whenever $S$ pressed the bar the tip of the tube was presented through the hole for $2 \mathrm{sec}$ so that $\mathrm{S}$ could lick the tube by extending his tongue through the hole. During the 2-sec dipper presentation barpresses were recorded and did not operate the dipper.

Events were programmed and recorded automatically by a BRS solid-state system located in the experimental room. Barpresses and the number of dipper presentations were recorded by means of counters. The session duration and latency of barpressing were recorded by timers as the number of pulses occurring at the rate of one every $15 \mathrm{sec}$ and one per second, respectively.

Procedure
Levels of sucrose and saccharin were selected from the literature. Guttman (1953) reported maximum and minimum barpressing to a $16 \%$ and $4 \%$ sucrose solution, respectively. Collier \& Novell (1967) reported that maximum daily intake of saccharin solution was at $.10 \%$; minimum intake was between $1.5 \%$ and $2.7 \%$. On the basis of a pilot study, it was determined that a $.10 \%$ saccharin solution produced a rate of barpressing equivalent to that of a $16 \%$ sucrose solution and that 


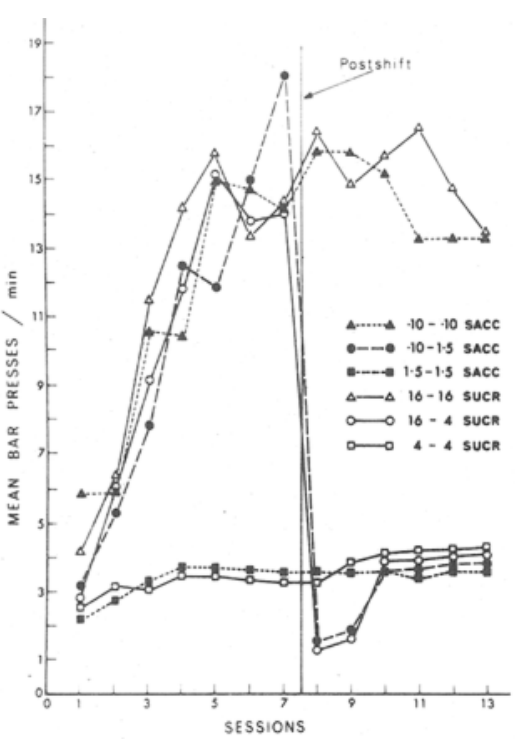

Fig. 1. Mean barpresses per minute for saccharin and sucrose.

a $1.5 \%$ saccharin solution produced a rate of barpressing similar to that of a $4 \%$ sucrose solution.

Five Ss were assigned randomly to each of six groups. Sucrose concentration treatments of $4 \%$ and $16 \%$ by weight ${ }^{1}$ and saccharin concentration treatments of $1.5 \%$ and $.10 \%$ by weight were used with two control groups, two shift groups, and two unshifted groups. The $4 \%$ control group $(4 / 4-C)$ and the $1.5 \%$ control group $(1.5 / 1.5-\mathrm{C})$ were run throughout the experiment without changing concentrations. Of the remaining groups, two were shifted after barpress asymptotes were reached and two were unshifted. The $16 / 4$ group was shifted on the eighth session from $16 \%$ sucrose to $4 \%$ sucrose, and the $.10 / 1.5 \%$ group was shifted on the eighth session from .10\% saccharin to $1.5 \%$ saccharin. The $16 / 16$ group and the $.10 / .10$ group remained unshifted after barpress asymptotes were reached.

Following several days of adaptation to a food- and water-deprivation schedule (Teklad pellets and water daily for $1 \mathrm{~h}$ at the end of the experimental time), Ss were trained on successive days to press the bar for continuous reinforcement (with each depression the bar operated the magazine). Each session lasted for 40 reinforcements (dipper presentations) This regime was continued until performance appeared to be stable (requiring seven sessions). Concentrations were then changed or not and all Ss were continued through the 13 th session. Throughout the study, Ss had access to dry pellets and water for $1 \mathrm{~h}$ following the experimental period.

Results

Five measures were examined in the analysis of the results: average number of barpresses made during each session, average volume (milliliters) of liquid consumed during each session, average latency of the 15th barpress per session, average barpresses per minute (number of barpresses in the session/session duration), and average consumption per minute (milliliters of liquid consumed in the session/session duration).

Since average barpresses per minute yielded essentially the same results as the above measures excluding average barpress latency, only average barpresses per minute are reported here. Figure 1 is illustrative of all measures.

From Fig. 1, it seems clear that in Sessions 1-7 $10 \%$ saccharin produced the same rate of barpressing as $16 \%$ sucrose, and $1.5 \%$ saccharin produced the same rate of barpressing as $4 \%$ sucrose. It seems equally clear that $.10 \%$ saccharin and $16 \%$ sucrose produced higher rates of barpressing than did $1.5 \%$ saccharin and $4 \%$ sucrose, respectively. The mean number of barpresses per minute in Sessions 1-7 differed significantly among the six groups by analysis of variance $(F=5.26$, $\mathrm{df}=5 / 24, \quad \mathrm{p}<.005)$. By Duncan's comparisons, the difference between $.10 / .10$ and $16 / 16, .10 / 1.5$ and $16 / 4$, and $1.5 / 1.5-\mathrm{C}$ and $4 / 4-\mathrm{C}$ was not statistically significant $(\mathrm{p}>.05)$, while the difference between the shift groups (.10/1.5 and $16 / 4)$ and the respective controls (1.5/1.5-C and 4/4-C) was highly reliable $(\mathrm{p}<.005)$.

Figure 1 indicates that on Session 8 the shift groups abruptly reduced their barpress rates to an equal level below their respective control groups (equal negative contrast effects). The mean number of barpresses per minute during Sessions 8-9 differed significantly among the two shift and the two control groups by analysis of variance $(F=6.03, \mathrm{df}=3 / 16, p<.01)$. By Duncan's comparisons, the difference between the shift groups was not significant $(p>.05)$, while the differences between the shift groups and their respective control groups were significant $(\mathrm{p}<.05)$. All measures showed these significant concentration differences in the preshift and negative contrast effects in the postshift.

\section{EXPERIMENT 2}

Experiment 1 indicated that $.10 \%$ and $1.5 \%$ are appropriate concentrations of saccharin solution to produce negative contrast effects. In Experiment 2 these concentrations were selected to examine the influence of a partial schedule of reinforcement on a shift in incentive magnitude in the relative absence of confounding factors.

Subjects, Apparatus, and Procedure

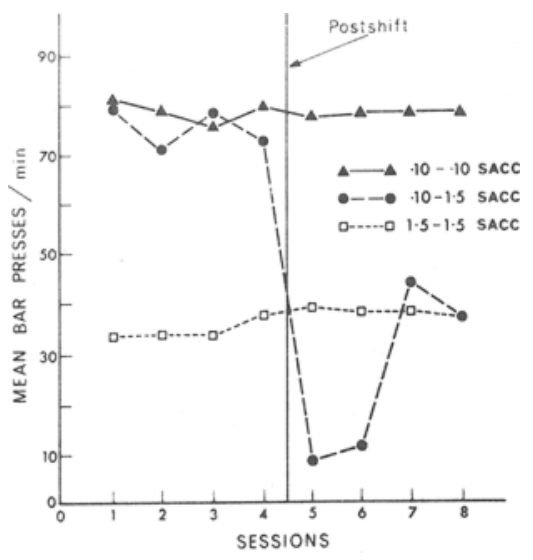

Fig. 2. Mean barpresses per minute for the eight FR 7 sessions with saccharin and partial reinforcement.

The Ss were 18 experimentally naive male hooded rats. The details of age, housing, and apparatus were the same as in Experiment 1, with the exception that the bar was set to operate the magazine on every seventh depression (FR 7). Six Ss were assigned randomly to each of three groups. Saccharin concentration treatments of $1.5 \%$ and $.10 \%$ by weight were used, with one control group, one shift group, and one unshifted group. In pretraining, all groups received five sessions of CRF followed by two sessions of FR 3. All groups then received eight sessions of FR 7. The $1.5 \%$ control group (1.5/1.5-C) and the unshifted group $(.10 / .10)$ were run throughout the experiment without changing concentrations. The .10/1.5 group was shifted on the fifth session after pretraining from .10\% to $1.5 \%$. The experiment was terminated for all groups at the end of the eighth session after pretraining.

\section{Results}

The same five measures of Experiment 1 were examined in Experiment 2. Mean barpresses per minute yielded essentially the same results as the other measures, excluding average barpress latency. Only average barpresses per minute for the eight FR 7 sessions are reported here. Figure 2 is representative of all measures except average barpress latency. From Fig. 2 it appears clear that in Sessions 1-4.10\% produced a higher rate of barpressing than did $1.5 \%$. The mean number of barpresses differed significantly among the three groups by analysis of variance $(F=6.91$, $\mathrm{df}=2 / 15, \mathrm{p}<.01)$. By Duncan's comparisons, the difference between $.10 / .10$ and $.10 / 1.5$ was not significant $(p>.05)$, while the difference between $.10 / .10$ and $1.5 / 1.5-C$, and between $.10 / 1.5$ and $1.5 / 1.5-\mathrm{C}$ was significant $(\mathrm{p}<.01)$.

Beginning with Session 5, concentration 
was changed for the shift group. Figure 2 shows that the shift group suddenly decreased its barpress rate to below the control group level (negative contrast effect). The average number of barpresses during Sessions 5-6 differed significantly between the shift and control group by analysis of variance $(F=14.65, d f=1 / 10$, $\mathrm{p}<.005$ ). All measures but latency showed a significant concentration difference in the preshift and negative contrast effects in the postshift.

\section{DISCUSSION}

Experiments 1 and 2 clearly demonstrated negative contrast effects with a change in concentration of a constant volume of saccharin solution. This result disagrees with Hulse (1962) and Vogel et al (1968). As already discussed, the discrepant findings may be due to the different preshift concentrations selected by the two studies previously cited and the present investigation. Since negative contrast effects are obtained with saccharin, the phenomenon cannot be explained on the basis of confoundings inherent in previous research, which has used solid food or sucrose as incentive.

Experiment 1 demonstrated negative contrast effects with a reduction in the concentration of a constant-volume sucrose solution. The finding is inconsistent with many previous reports in the literature. Rosen \& Ison (1965), Rosen (1966), and Ison \& Glass (1968) have reported no evidence of negative contrast effects using sucrose. The studies have used the consummatory response, and it may be difficult to compare these results with procedures that have used the (more traditional) barpress response. Collier \& Bolles (1968) have recently pointed out that burst rate (seven licks per second) is insensitive to changes in concentration of sucrose, while the interval between bursts is readily affected by changes in concentration. Therefore, changes in incentive magnitude would produce changes in lick rate proportional to the duration of the session. The barpress response does not contain a similar constant (burst rate), which under certain circumstances would render it insensitive to experimental manipulations.

The results of Experiment 2 indicate that negative contrast effects are obtained under a partial schedule of reinforcement in the relative absence of confounding factors.

\section{REFERENCES}

COLLIER, G. H., \& BOLLES, R. Some determinants of the intake of sucrose solutions. Journal of Comparative \& Physiological Psychology, 1968, 65, 379-383. COLLIER, G. H., \& NOVELL, K. Saccharine as a sugar surrogate. Journal of Comparative \&
Physiological Psychology. 1967, 64. 404-408. Di LOLLO. V. D.. \& BEEZ. V. Negative contrast effect as a function of magnitude of reward decrement. Psychonomic Science, 1966. 5. 99-100.

DLNHAII, P. J., \& KILPS, B. Shifts in magnitude of reinforcement: Confounded factors or contrast effects? Journal of Experimental Psychology, 1969, 79, 373-374.

HULSE, S. H. Partial reinforcement, continuous reinforcement, and reinforcement shift effects Joumal of Experimental Psychology, 1962, $64.451-459$

ISON, 3. R.. \& GLASS, D. H. Consummatory training and subsequent instrumental behavior: A long-term consequence of differential reinforcement magnitudes. Journal of Comparative \& Physiological Psychology, $1968,65,524-525$.
ROSEN, A, J. Incentive-shift performance as a function of inagnitude and number of sucrose rewards. Journal of Comparative \& Physiolosical Psychology, 1966, 62, 487-490.

ROSEN, A. J.. \& ISON, J. R. Runwaway performance following changes in sucrose rewards. Psychonomic Science, 1965, 2, 335-336.

VOGEI, J. R.. MIKULKA, P. J., \& SPEAR, N. E. Effects of shifts in sucrose and saccharine concentrations on licking behavior in the rat. Joumal of Comparative \& Physiological Psychology, 1968, 66, 661-666.

\section{NOTE}

1. For example, the $16 \%$ solution was prepared by mixing $16 \mathrm{~g}$ of cane sugar with $84 \mathrm{ml}$ of tap water. The saccharin solutions were prepared in a similar manner with U.S.P. granular saccharin.

\title{
Light deprivation and visual-cliff performance in the adult cat*
}

\author{
GWENYTH JONES and ROBERT PASNAK \\ The Catholic University of America, Washington, D.C. 20017
}

Eight adult cats were light deprived for 80 days and tested on the visual cliff. No deficit in performance was found on this measure of depth perception. Species differences and implications for explanations in terms of visual activity and critical maturation periods were discussed.

The young of many species have been

*Special thanks are extended to Dr. William Pare and his staff at the Veterans Administration Hospital, Perry Point, Maryland, for their generous help and support. This project was funded by PHS Grant FRO 7123-01. dark reared and typically show both behavioral and physiological deficits (see Beach \& Jaynes, 1954). For example, kittens show a deficit in depth perception when tested on the visual cliff after 27 days of dark rearing (Walk \& Gibson, 